\title{
Linking the YTH domain to cancer: the importance of YTH family proteins in epigenetics
}

\author{
Rongkai Shi', Shilong Ying', Yadan Li', Liyuan Zhu (1)', Xian Wang² and Hongchuan Jin
}

\begin{abstract}
N6-methyladenosine (m6A), the most prevalent and reversible modification of mRNA in mammalian cells, has recently been extensively studied in epigenetic regulation. YTH family proteins, whose YTH domain can recognize and bind m6Acontaining RNA, are the main "readers" of m6A modification. YTH family proteins perform different functions to determine the metabolic fate of m6A-modified RNA. The crystal structure of the YTH domain has been completely resolved, highlighting the important roles of several conserved residues of the YTH domain in the specific recognition of m6Amodified RNAs. Upstream and downstream targets have been successively revealed in different cancer types and the role of YTH family proteins has been emphasized in m6A research. This review describes the regulation of RNAs by YTH family proteins, the structural features of the YTH domain, and the connections of YTH family proteins with human cancers.
\end{abstract}

\section{Facts}

- The effects of YTH proteins on RNA metabolism are different but overlap to some degree.

- The structure of the YTH domain helps YTHDF proteins recognize and bind m6A-containing transcripts.

- Structural crystallography studies have elaborated the molecular basis of YTH domains to read m6Amodified RNA.

- YTH proteins have different targets in different cancers and are involved in almost every aspect of tumorigenesis and cancer progression.

\section{Open questions}

- What is the precise unified model of YTH proteins in the regulation of m6A modification? Are these proteins different or redundant?

\footnotetext{
Correspondence: Hongchuan Jin (jinhc@zju.edu.cn)

'Laboratory of Cancer Biology, Key Lab of Biotherapy in Zhejiang, Sir Run Run

Shaw Hospital, Cancer Center, Zhejiang University, Hangzhou, China

${ }^{2}$ Department of Medical Oncology, Sir Run Run Shaw Hospital, Medical School

of Zhejiang University, Hangzhou, China

These authors contributed equally: Rongkai Shi, Shilong Ying

Edited by F. Pentimalli
}

- Is evaluating structural differences in YTH domains the potential direction for exploring and explaining the complex phenomena in this field?

- Is a highly selective YTH domain inhibitor a potential therapeutic agent for cancer?

- Is the existing structural information about the YTH domain useful for guiding the rational design of selective YTH domain inhibitors?

\section{Introduction}

Since the 1950s, nucleotides, which are the basic molecular components of RNA, have been found to undergo a number of chemical modifications on their adenosine $(\mathrm{A})$, guanosine $(\mathrm{G})$, cytidine $(\mathrm{C})$, and uridine (U) nucleosides. Over 100 kinds of RNA modifications, such as $\mathrm{hm} 5 \mathrm{C}, \mathrm{m} 1 \mathrm{~A}$, and $\mathrm{m} 6 \mathrm{~A}$, have been found and these modifications can affect the biogenesis, structure, and function of RNA in different ways; these modifications have been the hotspots in epigenetics in recent years and still have great potential for exploration. N6-methyladenosine (m6A) has been considered the most prevalent and reversible modification of mRNA in eukaryotic cells since its initial discovery in the $1970 \mathrm{~s}^{1}$. m6A modification is generally located in the consensus motif DRACH $(D=G, A$, or $U ; R=G$ or $A$; 
$\mathrm{H}=\mathrm{A}, \mathrm{C}$, or $\mathrm{U})$, which is enriched in the coding sequence (CDS) and $3^{\prime}$ untranslated region ( $3^{\prime}$ UTR) of $\mathrm{RNA}^{1-4}$. The regulation of m6A depends on three important factors, "writers", "erasers", and "readers". "Writers" and "erasers" add and remove m6A modifications to and from RNA, respectively, while "readers" recognize m6A and affect the fate of RNA. Generally, m6A reader proteins can be divided into three classes, which use the YTH domain (YTH family proteins), m6A switch mechanism (hnRNPC, hnRNPG, and hnRNPA2B1), or common RNA-binding domain and its flanking regions (IGF2BPs and hnRNPA2B1) to bind m6A-containing transcripts ${ }^{5}$. In recent years, writers and erasers have been actively researched ${ }^{6}$, and recently, the focus has gradually turned to readers, especially YTH family proteins, due to the application of several novel methods. m6A-seq, based on antibodymediated capture and massively parallel sequencing, helps us identify m6A sites at the transcriptome level. In addition to traditional methods such as RIP-seq, new techniques such as CLIP-seq and PAR-CLIP, based on combining immunoprecipitation with in vivo UV crosslinking enhanced by photoactivatable ribonucleosides, help us identify the targets of m6A readers ${ }^{7-10}$. These methods are shedding new light on RNA modifications. Therefore, we attempt to review the regulation of transcripts by YTH family proteins, the structural basis of the YTH domain, and the association of YTH family proteins with human cancers.

\section{YTH family proteins recognize $\mathrm{m} 6 \mathrm{~A}$ and regulate RNA processes}

m6A modification is regulated by RNA methyltransferase complexes-writers-and demethylaseserasers. To catalyze N6-methyladenosine (m6A) RNA methylation, methyltransferase-like 3 (METTL3) and human methyltransferase-like 14 (METTL14) form a stable heterodimer core complex with Wilms tumor 1associated protein (WTAP), which enables the complex to localize to nuclear speckles enriched with pre-mRNA processing factors ${ }^{11,12}$. Additionally, other adaptor proteins, such as RBM15/15B, VIRMA, and ZC3H13, have been shown to be important for facilitating the function of the methyltransferase complex ${ }^{13-15}$. Regarding demethylases, only fat mass and obesityassociated (FTO) and AlkB homolog 5 (ALKBH5) have been found to be available to catalyze m6A demethylation thus far ${ }^{16,17}$. The YTH domain, the structural basis for the recognition and binding of m6A-modified RNA, enables a series of proteins such as YTHDF1-3 and YTHDC1-2 to act as readers in the regulation of m6Acontaining transcripts. Different YTH family proteins function in different ways to influence RNA splicing, export, translation, and decay (Fig. 1).

\section{YTHDF1-3}

YTHDF1 selectively recognizes m6A-modified mRNAs via the YTH domain, promotes their loading into the ribosome, and interacts with initiation factors to facilitate their translation via the $\mathrm{N}$-terminal domain ${ }^{18}$. In addition to mediating translation initiation, YTHDF1 can also bind to the m6A site in the CDS of some mRNAs to assist with translation elongation ${ }^{19}$. Additionally, some research has indicated that YTHDF1 may sometimes mediate the target transcript's stability ${ }^{20-22}$. It is worth mentioning that YTHDF1 is of great importance in the field of neuroscience since it was reported to be capable of inducing axon regeneration ${ }^{23}$, regulating axon guidance $^{24}$, and thereby facilitating learning and memory ${ }^{25}$ by enhancing the translation of specific transcripts. Conversely, YTHDF2 selectively binds m6A-modified RNA and regulates its degradation by recruiting the CCR4-NOT complex to accelerate RNA deadenylation ${ }^{10,26,27}$. Another YTHDF2-mediated RNA degradation mechanism is endoribonucleolytic cleavage via HRSP12-mediated bridging of YTHDF2-bound RNAs to RNase P/MRP, through which a subset of circular RNAs is selectively downregulated in an m6A-dependent manner ${ }^{28}$. The $\mathrm{P} /$ $\mathrm{Q} / \mathrm{N}$-rich N-terminal domain of YTHDF2 is responsible for its function in RNA decay, while its aa 101-200 region interacts with the SH domain of CNOT1, and its aa 1-100 region is the HRSP12 binding region. In addition, YTHDF3 interacts with YTHDF1 to help promote protein translation, and with YTHDF2 to affect the decay of methylated mRNA transcripts ${ }^{29}$. There is also a model of m6A regulation in which pre-mRNA is first recognized by YTHDF3, which acts as an assigner, and YTHDF1 and YTHDF2 then competitively interact with YTHDF3, thus determining the fate of the mRNA transcript ${ }^{30}$. Importantly, recent studies have revealed that YTHDFs can be involved in liquid-liquid phase separation by binding multi-m6A-modified mRNA scaffolds and then forming YTHDF-m6A-mRNA complexes. These complexes then partition into phase-separated compartments such as $\mathrm{P}$ bodies, stress granules, or neuronal RNA granules to decide whether the mRNA should be degraded, translated, or undergo other events. Especially in stress granule formation, YTHDF proteins are critical in recruiting mRNA to stress granules. In addition, this phase separation is significantly enhanced by mRNAs that contain multiple m6A motifs. In contrast to mRNAs containing a single m6A site, mRNAs containing multiple m6A sites tend to act as scaffolds for the binding of YTHDF proteins to juxtapose their low-complexity domains and initiate phase separation ${ }^{31-34}$. Remarkably, given that YTH domain-containing proteins are $\mathrm{m} 6 \mathrm{~A}$ readers, it has also been reported that YTHDF2 and YTHDF3 can influence m6A modification levels by blocking the demethylase activity of FTO and ALKBH in different ways, for 


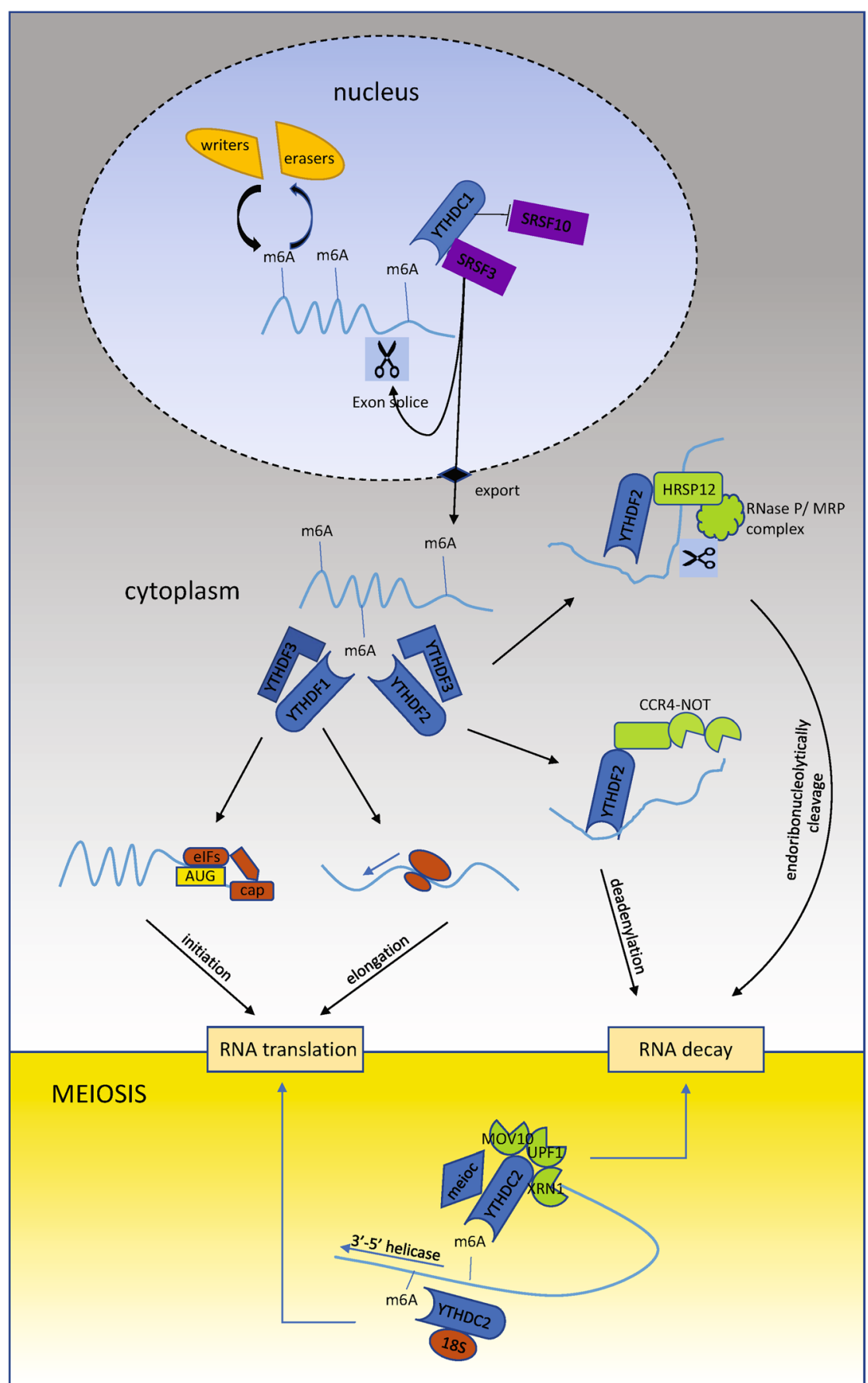

Fig. 1 Model of YTH family proteins modulating m6A-containing RNAs. In cell nucleus, "writers" and "erasers" add and remove m6A modifications to and from RNA. YTHDC1 regulates splicing and mediates the export of m6A-containing mRNAs by recruiting SRSF3, while blocking SRSF10 mRNA binding. In cytoplasm, YTHDF1 recognizes m6A-containing mRNAs and promotes its translation initiation and elongation. And the m6A-containing mRNAs can also be recognized by YTHDF2, which promotes their degradation through two pathways, CCR4-NOT complexmediated deadenylation and HRSP12-mediated endoribonucleolytic cleavage. YTHDF3 interacts with YTHDF1 and YTHDF2 to accelerate metabolism of m6A-modified mRNAs. YTHDC2 mainly functions to regulate the switch from mitosis to meiosis by interacting with MEIOC. YTHDC2 interacts with XRN1, UPF1, and MOV10 to destabilize its target RNAs. Also, the binding of YTHDC2 to the 185 rRNA and its $3^{\prime}-5^{\prime}$ RNA helicase activity facilitates the translation of its target RNAs. 
instance, in heat shock stress-induced transcripts ${ }^{35-37}$. However, recently, some researchers developed a fundamentally different model to explain the effect of YTHDF proteins on m6A-containing transcripts. They hypothesized that the function of YTHDF proteins is redundant and that each paralog compensates for the function of the other paralogs. More importantly, YTHDF proteins show identical binding to all m6A-containing transcripts and function together to promote the decay of these transcripts $^{38-40}$.

\section{YTHDC1-2}

YTHDC1, also called YT521-B, was first found to be an RNA splicing-related protein in 1988 because of its glutamic acid/arginine-rich carboxy domain with splicing factors $^{41}$. It is a ubiquitously expressed nuclear protein and localized in YT bodies adjacent to nuclear speckles ${ }^{42}$. It interacts with SAF-B and Sam68 to modulate alternative splice site selection in a concentration-dependent manner, which is regulated by tyrosine phosphorylation mediated by src kinases ${ }^{43}$. Additionally, YTHDC1 shuttles between the nucleus and the cytosol, where it is phosphorylated by SRC and TEC tyrosine kinase family members, causing its transformed function in RNA splicing $^{44}$. Recently, as the ability of the YTH domain to recognize $\mathrm{m} 6 \mathrm{~A}$ has gradually been recognized, the association between YTHDC1 and m6A has been partially clarified. YTHDC1 promotes exon inclusion in m6Amodified RNA transcripts in an m6A-dependent manner by recruiting the pre-mRNA splicing factor SRSF3 while blocking SRSF10 mRNA binding to regulate splicing ${ }^{45}$. The interaction with SRSF3 helps YTHDC1 steer target mRNAs into the mRNA nuclear export pathway; thus, YTHDC1 mediates the export of m6A-containing mRNAs $^{46}$. For instance, YTHDC1 mediates m6Adependent mRNA splicing to control neuronal functions and fine-tune sex determination in Drosophila ${ }^{47,48}$. In addition to playing roles in mRNA splicing and export, YTHDC1 was recently found to regulate the stability of mRNA. YTHDC1 facilitates the decay of chromosomeassociated regulatory RNAs in an m6A-dependent manner through nuclear exosome targeting-mediated nuclear degradation and thus decreases chromatin accessibility and represses gene expression ${ }^{49}$. Furthermore, YTHDC1 is involved in controlling the stability of MAT2A mRNA, which is upregulated through mRNA stabilization in response to $S$-adenosylmethionine depletion ${ }^{50}$. Importantly, YTHDC1 recognizes m6A residues on XIST, which is essential for XIST's function in mediating the transcriptional silencing of genes on the $\mathrm{X}$ chromosome ${ }^{13}$. However, the mechanism by which YTHDC1 binding to XIST leads to gene silencing remains unclear. In addition to its function as an RNA-binding protein, YTHDC1 promotes $\mathrm{H} 3 \mathrm{~K} 9 \mathrm{me} 2$ demethylation and gene expression by interacting with and recruiting KDM3B to chromatin regions ${ }^{51}$. The molecular role of the last member of the YTH family, YTHDC2, in the regulation of m6A remains uncertain. YTHDC2 was first found to be an RNA helicase with a YTH domain ${ }^{52}$. Similar to other YTH family proteins, YTHDC2 is able to recognize and bind m6A moieties in mRNA to have a regulatory role ${ }^{53,54}$. Several studies have revealed the importance of YTHDC2 in the meiosis of germline cells. YTHDC2 interacts with MEIOC, a meiosis-specific protein, and XRN1, a $5^{\prime}-3^{\prime}$ exoribonuclease, to regulate the switch from mitosis to meiosis through posttranscriptional regulation of target transcripts ${ }^{54-58}$. YTHDC2 enhances the translation efficiency of its targets and decreases their mRNA abundance $^{53}$. It is an RNA-induced ATPase with $3^{\prime}-5^{\prime}$ RNA helicase activity mediated by its DEAH helicase domain ${ }^{54}$. The ankyrin repeats help YTHDC2 to interact with XRN1, UPF1, and MOV10, probably to destabilize its target RNAs ${ }^{53,54,59}$. The binding of YTHDC2 to the $18 \mathrm{~S}$ rRNA of the small ribosomal subunit in close proximity to the mRNA entry tunnel and exit site suggests that YTHDC2 directly bridges interactions between m6Acontaining mRNAs and the ribosome to promote translation $^{59}$. It has also been implied that the $\mathrm{R} 3 \mathrm{H}$ domain contributes to RNA binding by stabilizing the interaction between YTHDC2 and its m6A-containing substrates ${ }^{59}$. Interestingly, m6A residues located within the coding sequence (CDS) positively regulate translation by resolving mRNA secondary structures, for which YTHDC2 is required because of its helicase activity ${ }^{60}$. Recently, two hepatic studies also provided evidence that YTHDC2 helps decrease the stability of its target mRNAs and inhibit gene expression in an m6A-dependent manner ${ }^{61,62}$.

\section{Structural basis for the selective binding of $\mathrm{m} 6 \mathrm{~A}-$ modified RNA by YTH family proteins}

After YT521-B was identified as an RNA splicingrelated protein, a conserved region in its protein sequence that is present only in eukaryotic genomes was identified and termed the YT homology (YTH) domain ${ }^{63}$. The YTH domain has been identified in 174 different proteins of eukaryotic species and is abundant in plants. The YTH domain contains 100-150 amino acids and is characterized by 14 invariant and 19 highly conserved residues ${ }^{64}$. In addition to humans, the YTH domain has also been found in Drosophila ${ }^{63}$, fission yeast ${ }^{65}$, Saccharomyces cerevisiae $^{66}$, Plasmodium falciparum $^{67}$, and many species of plants ${ }^{68-70}$. YTH domain-containing proteins in other eukaryotic species perform many of the same or similar functions as those found in humans. For example, the m6A mRNA methylation program has been revealed in the malaria parasite, and PfYTH1 and PfYTH2, YTH domain-containing proteins in $P$. falciparum, were 
confirmed to be m6A readers ${ }^{67,71}$. In Arabidopsis, m6A recognition by the YTH domain-containing proteins ECT2, ECT3, and ECT4 are important for cell proliferation and plant organogenesis ${ }^{72,73}$. In fission yeast, Mmil, a deeply researched YTH domain-containing protein, selectively recognizes a cis-acting region (DSR) specific for meiotic transcripts and directs them to the nuclear exosome for degradation $^{65,74}$. Mmil also directs RNAi-dependent heterochromatin formation and gene silencing through recruitment of Red1, the histone H3K9 methyltransferase Clr4/SUV39h, the RNA-induced transcriptional silencing (RITS) RNAi complex, and the conserved cleavage and polyadenylation factor $(\mathrm{CPF})^{75-78}$. The role of Mmil in affecting chromatin accessibility and heterochromatin is similar to that of its human homolog YTHDC1.

The presence of a YTH domain defines a group of proteins that includes YTHDF1-3, YTHDC1, and YTHDC2 in humans (Fig. 2A). After the YTH domain was first found to be able to bind single-stranded RNA, these five proteins were successively identified as m6A readers $^{4,18,53,64,79}$. Through NMR spectroscopy and X-ray diffraction analysis, the solution and crystal structures of the YTH domains of distinct YTH family members and their complexes with m6A RNA oligoribonucleotides have been solved successively ${ }^{80,81}$. Overall, these YTH domains commonly adopt a specific mixed $\alpha$-helix$\beta$-sheet fold in which the $\beta$-sheets are arranged into a central, globular $\beta$-barrel fold and surrounded by $\alpha$-helices, providing a hydrophobic core where several highly conserved aromatic residues are located. Electrostatic potential analysis of the protein surface demonstrated that the surface of the YTH domain has a positively charged concave structure, which is enriched with basic residues such as lysines and arginines and is responsible for RNA binding. Intriguingly, a well-defined conserved aromatic cage is observed in all YTH domains, which endows the YTH domain with the capacity for discriminative recognition of N6-methyladenosine-modified RNA (Fig. 2B).

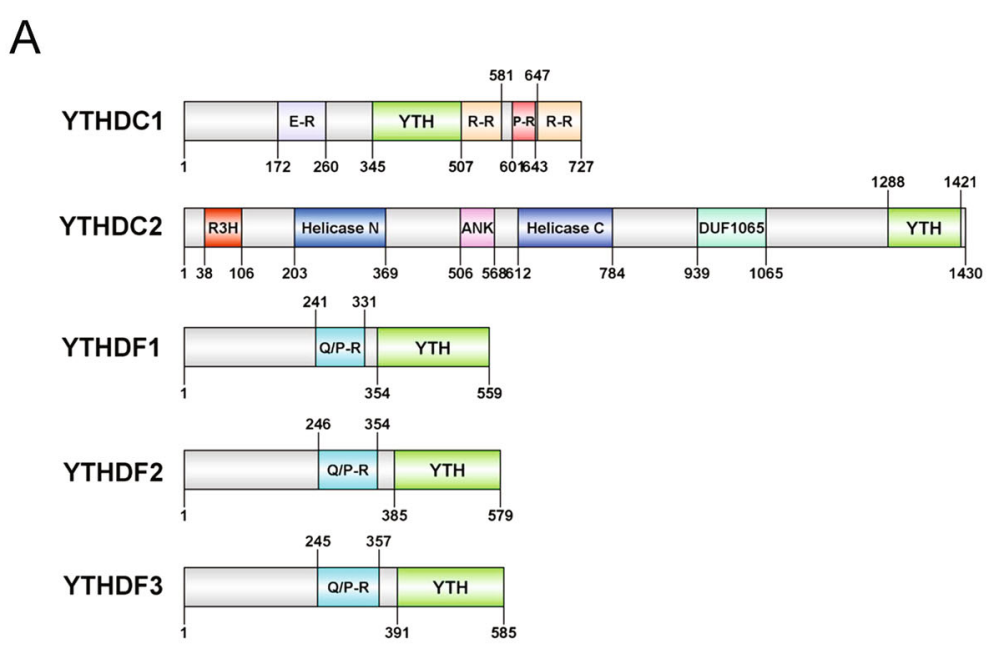

B

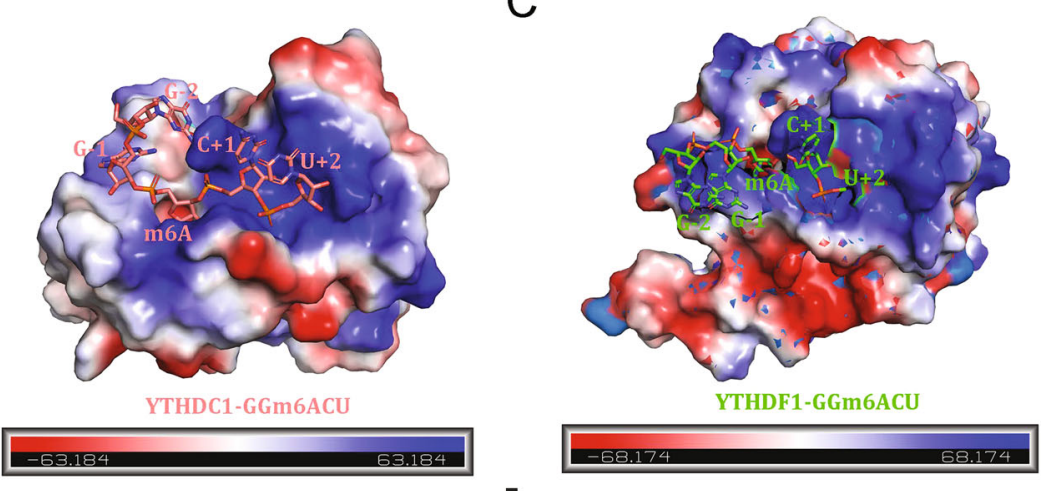

Fig. 2 The YTH domain is usually located in the $C$ terminus of proteins and is rich in basic amino acid residues for RNA binding. A Schematic representation of the protein structure of human YTH domain-containing proteins (YTHDC1, YTHDC2, YTHDF1, YTHDF2, and YTHDF3). B, C The electrostatic potential surface of the YTH domains of YTHDC1 and YTHDF1 in complex with m6A-containing oligonucleotides is represented by PyMOL 2.0. Positive charges are colored blue, neutral charges are white, and negative charges are red. 

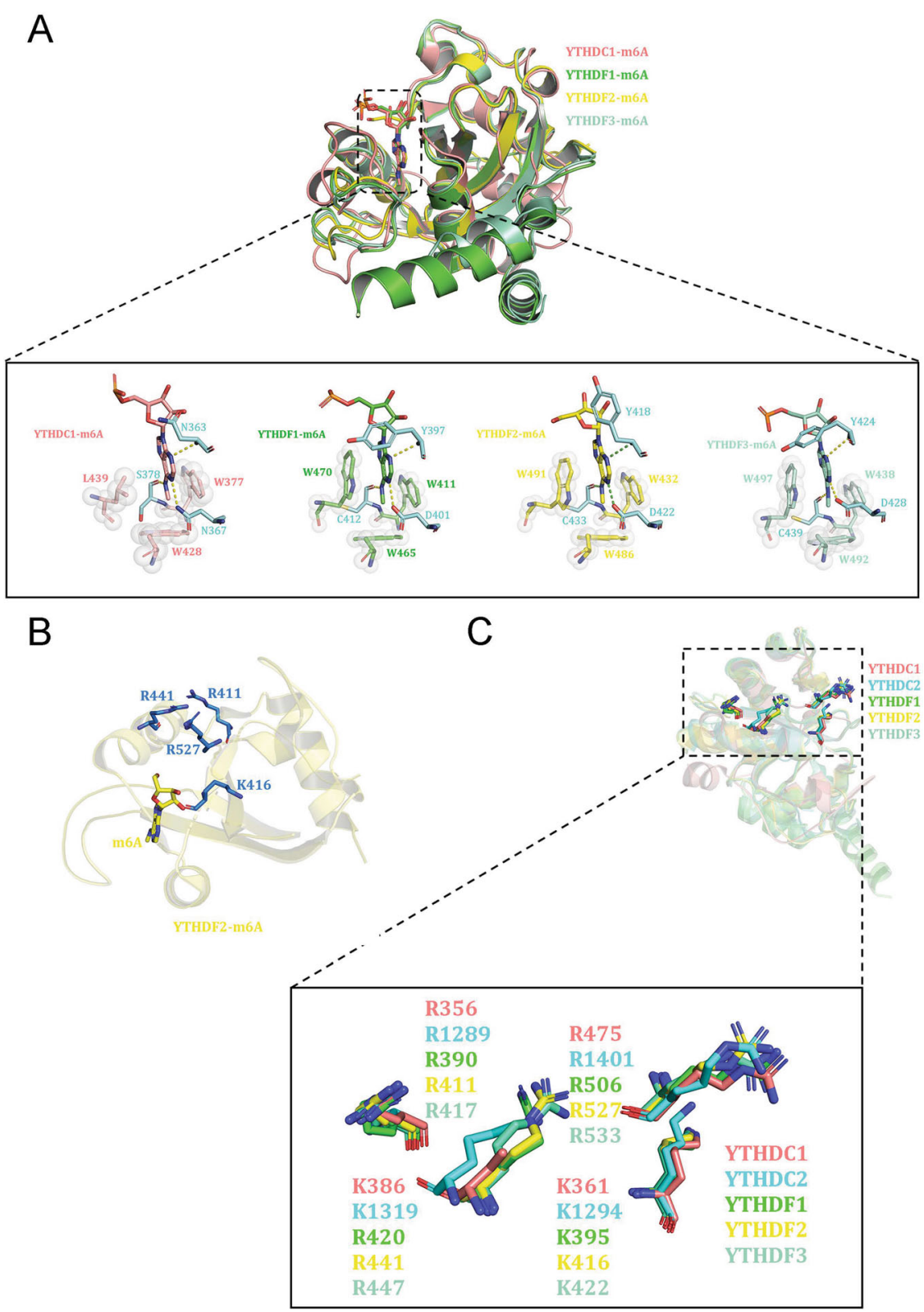

Fig. 3 The conserved aromatic residues and basic residues in the YTH domain are responsible for methylated adenosine (m6A) recognition and binding, respectively. A Superposition of the complex structures of the YTHDC1 YTH domain (YTH-YTHDC1)-m6A (pink), YTHYTHDF1-m6A (green), YTH-YTHDF2-m6A (yellow), and YTH-YTHDF3-m6A (pale green), showing the conserved aromatic cage for specific recognition of the methyl group of m6A. Both the residues in the aromatic cage of YTHDC1 and the 6-methylated adenine base (m6A) are shown in the model as pink sticks, and the counterparts in YTHDF1, YTHDF2, and YTHDF3 are shown in the model as green, yellow, and pale green sticks, respectively. In addition, the adenine base always forms three hydrogen bonds with adjacent residues (cyan sticks), which is essential for the binding between m6A and YTH domains. The hydrogen bonds are labeled with dashed lines. The corresponding PDB IDs are 4R3I, 4RCJ, 4RDN, and 6ZOT. B Four basic residues, R411, K416, R441, and R527, on the surface of the YTHDF2 YTH domain, are critical for binding to the RNA backbone. C Superposition of the YTH domains of YTHDC1 (pink cartoon), YTHDC2 (cyan cartoon), YTHDF1 (green cartoon), YTHDF2 (yellow cartoon), and YTHDF3 (pale green cartoon). The four basic residues are highly conserved in these human YTH proteins. The basic residues R411, K416, R441, and R527 in YTHDF2 and their corresponding residues in the YTH domains of YTHDC1, YTHDC2, YTHDF1, and YTHDF3 are shown as yellow, pink, cyan, green, and pale green sticks.

The first aromatic cage was discovered in the YTH domain of YTHDC1 and consists of two aromatic tryptophans (W377 and W428) and an atypical nonaromatic leucine (L439), which forms vital $\pi-\pi$ interactions with the adenine base and hydrophobic interactions with the N6methyl moiety ${ }^{79}$ (Fig. 3A). Mutation of either W377 or W428 abolishes the binding of YTHDC1 to m6A, highlighting its critical role in specifically recognizing N6- 
methylated adenine bases. In addition to the above fundamental hydrophobic interactions, the $\mathrm{N}$ atoms in the methyladenine base (N6, N3, and N1) form hydrogen bonds with three residues (S378, N363, and N367) of YTHDC1 adjacent to the cage, making the binding between YTHDC1 and m6A more stable (Fig. 3A). Interestingly, Xu et al. found that when N367 is mutated to D367, m6A binding of YTHDC1 is abolished. Considering the difference in the protonation state between N367 and D367, it is predicted that the N1 atom is in a deprotonated state and thus would preferentially bind to deprotonated N367 over protonated D367 ${ }^{82}$. In addition, the detailed structural analysis further specified that the basic residues around the aromatic cage also make significant contributions to the binding affinity of YTHDC1 for m6A-modified RNA. For example, the cytidine in GG (m6A)CU not only forms one hydrogen bond with the side chain of R475 but is also stacked with the guanidinium group of R475 and the adjacent uracil through cation $-\pi$ and $\pi-\pi$ interactions, respectively. Mutating R475 to phenylalanine or an alanine reduces the binding affinity by 9 -fold or over 100 -fold, respectively, suggesting the importance of this residue in maintaining YTHDC1RNA binding. Recently, the crystal structure of the YTHDC2 YTH domain was solved and compared with that of the YTHDC1 domain in complex with GG(m6A) $\mathrm{CU}$ oligonucleotides ${ }^{83}$. Similarly, the YTH domain of YTHDC2 contains three aromatic residues (W1310, W1360, and L1365) corresponding respectively to W377, W428, and L439 of YTHDC1, which constitute a conserved hydrophobic pocket for m6A recognition. Furthermore, a positively charged surface around the m6A binding pocket is also observed in the YTHDC2 YTH domain, indicating that YTHDC2 adopts an architecture similar to that of YTHDC1 to accommodate m6A RNA moieties.

With the clarification of the YTHDC1-m6A complex structure, the structures of three cytoplasmic m6A readers, YTHDF1, YTHDF2, and YTHDF3, in complex with m6A-modified RNA have successively been solved by three individual groups ${ }^{40,82,84}$. Unsurprisingly, the YTH domains of YTHDF1, YTHDF2, and YTHDF3 harbor a specific m6A RNA-binding surface and aromatic cage similar to that of YTHDC1; this cage is composed of W411, W465, and W470 in YTHDF1; W432, W486, and W491 in YTHDF2; and W438, W492, and W497 in YTHDF3 (Fig. 3A). Mutating the above tryptophans to alanines drastically impairs the binding of YTHDF1 and YTHDF2 to m6A RNA. Additionally, three conserved residues in YTHDF1 (C412, Y397, and D401), YTHDF2 (C433, Y418, and D422), and YTHDF3 (C439, Y424, and D428) that are located near the aromatic cage and correspond to S378, N363, and N367 in YTHDC1 form hydrogen bonds with the N6 amino group in m6A, N3 in purine rings, and $\mathrm{N} 1$ in purine rings, respectively (Fig. 3A). Mutation of Y397 in YTHDF1 results in a marked decline in its m6A RNA binding, suggesting that these hydrogen bonds are also of great importance in m6A recognition. Of particular note is that N367 in YTHDC1 is substituted for D401 in YTHDF1, which may explain why the binding affinity of YTHDC1 for GG(m6A)CU $\left(K_{\mathrm{d}}=\right.$ $2.0 \mu \mathrm{M})$ is ten times higher than that of YTHDF1 $\left(K_{\mathrm{d}}=\right.$ $22.0 \mu \mathrm{M})^{82}$. Mutating D401 to N401 significantly enhances the binding capacity of YTHDF1, rendering it identical to that of YTHDC1 $\left(K_{\mathrm{d}}=1.5 \mu \mathrm{M}\right)$, confirming the considerable contribution of this specific residue to $\mathrm{m} 6 \mathrm{~A}$ RNA binding. Moreover, a basic patch composed of four basic residues, R411, K416, R441, and R527, is observed on the electrostatic potential surface of the YTHDF2 YTH domain $^{85}$ (Fig. 3B). Sequence alignment of the five YTH domains showed that these four residues are almost completely conserved in YTHDF1-3 and YTHDC1-2 (Fig. $3 \mathrm{C})$. The results of mutagenesis experiments demonstrated that mutating K416 and R527 of YTHDF2 to alanines not only heavily reduces the m6A binding affinity of YTHDF2 by approximately 25 -fold but also decreases the binding of unmethylated RNA (A-RNA) by over 5fold and 10-fold, respectively. Similarly, the binding affinity of the YTHDF2 R411A mutant for m6A RNA and ARNA is decreased by $\sim 3$-fold and 2-fold, respectively, revealing that the basic patch on the surface of the YTH domain has a dominant role in binding to the RNA backbone but probably does not participate in m6A recognition. To more clearly delineate the molecular mechanisms underlying the specific recognition of m6Amodified RNA by human YTH family proteins, a summary of binding affinities of the wild-type and YTH domain mutants of YTHDC1, YTHDF1, and YTHDF2 to 5-mer and 17-mer m6A-modified RNA oligonucleotides are provided in Table 1.

A previous study of the m6A RNA methylome by borate gel chromatography suggested that m6A modification is highly enriched in RRACU (where R is A or G) sequences in mammals $^{86}$. Recent PAR-CLIP studies on transcriptome-wide YTHDC1 binding sites also identified $\mathrm{GG}(\mathrm{m} 6 \mathrm{~A}) \mathrm{C}$ as the highest affinity binding motif of $\mathrm{YTHDC1}^{79}$. Furthermore, quantitative isothermal titration calorimetry (ITC) binding assay confirmed that the binding affinity of YTHDC1 for GG(m6A)CU $\left(K_{\mathrm{d}}=\right.$ $2.0 \mu \mathrm{M})$ was severely impaired when the second $\mathrm{G}$ nucleotide (G-1) was replaced with A (A-1, $\left.K_{\mathrm{d}}=15.0 \mu \mathrm{M}\right)$. However, subsequent comprehensive analysis of the sequence preference of different YTH domains for m6A RNAs revealed that no YTH domain except for that in YTHDC1 exhibits sequence selectivity at the position preceding the m6A modification $^{82}$ (Table 2). By superposition of the crystal structures of the YTHDF1-GG (m6A)CU and YTHDC1-GG(m6A)CU complexes, several 
Table 1 Binding affinities of the wild-type and mutant YTH domains of YTHDC1, YTHDF1, and YTHDF2 for m6Amodified RNAs.

\begin{tabular}{|c|c|c|c|}
\hline RNA sequence & Proteins & Genotype & $K_{\mathrm{d}}(\mu \mathrm{M})$ \\
\hline \multirow[t]{6}{*}{$\mathrm{GG}(\mathrm{m} 6 \mathrm{~A}) \mathrm{CU}$} & YTHDC1 & WT & $2.0 \pm 0.1$ \\
\hline & & W377A & NB \\
\hline & & W428A & NB \\
\hline & & R475F & $18 \pm 2$ \\
\hline & & R475A & $210 \pm 20$ \\
\hline & & N376D & $>100$ \\
\hline \multirow[t]{7}{*}{$\mathrm{GG}(\mathrm{m} 6 \mathrm{~A}) \mathrm{CU}$} & YTHDF1 & WT & $22 \pm 4$ \\
\hline & & W411A & NB \\
\hline & & W465A & NB \\
\hline & & W470A & NB \\
\hline & & D401N & $1.5 \pm 0.1$ \\
\hline & & Y397A & NB \\
\hline & & $\mathrm{R} 506 \mathrm{~A}$ & $>200$ \\
\hline UUCUUCUGU & YTHDF2 & WT & 2.54 \\
\hline \multirow[t]{6}{*}{ GG(m6A)CUGUG } & & W432A & 16.64 \\
\hline & & W486A & 17.61 \\
\hline & & $\mathrm{K} 416 \mathrm{~A}$ & 62.75 \\
\hline & & R527A & 62.94 \\
\hline & & R411A & 7.81 \\
\hline & & R441A & 7.35 \\
\hline
\end{tabular}

NB no binding.

Table 2 Binding affinities of four human YTH family members (YTHDC1, YTHDC2, YTHDF1, and YTHDF2) to a 9mer methylated RNA oligonucleotide.

\begin{tabular}{|c|c|c|c|c|}
\hline \multirow[t]{2}{*}{ RNA sequence } & \multicolumn{4}{|l|}{$K_{\mathrm{d}}(\mu \mathrm{M})$} \\
\hline & YTHDC1 & YTHDC2 & YTHDF1 & YTHDF2 \\
\hline $\mathrm{CCG} \mathbf{A}(\mathrm{m} 6 \mathrm{~A}) \mathrm{CUGU}$ & $1.0 \pm 0.1$ & $24 \pm 2$ & $1.1 \pm 0.2$ & $0.9 \pm 0.2$ \\
\hline CCGG(m6A)CUGU & $0.22 \pm 0.03$ & $12 \pm 2$ & $0.8 \pm 0.3$ & $0.9 \pm 0.1$ \\
\hline $\mathrm{CCGC}(\mathrm{m} 6 \mathrm{~A}) \mathrm{CUGU}$ & $0.32 \pm 0.03$ & $14 \pm 1$ & $0.8 \pm 0.2$ & $0.7 \pm 0.2$ \\
\hline $\mathrm{CCGU}(\mathrm{m} 6 \mathrm{~A}) \mathrm{CUGU}$ & $0.30 \pm 0.06$ & $16 \pm 3$ & $0.9 \pm 0.2$ & $0.8 \pm 0.2$ \\
\hline
\end{tabular}

The nucleotide preceding the m6A mark is highlighted in bold to depict the binding preference of the four YTH proteins for different m6A-containing RNAs.

key structural divergences were observed that may reasonably explain the unique nucleotide selectivity of YTHDC1 at the -1 position. First, G-1 (the G nucleotide preceding the m6A) forms two hydrogen bonds with V382 and N383 and interacts with L380 and M438 by hydrophobic interactions (Fig. 4), which might be abolished by replacing G-1 with A-1. Second, L380 and M438 are exclusive to YTHDC1, while their counterparts in other YTH proteins are polar amino acids, for example, T414 and K469 in YTHDF1 and T435 and K490 in YTHDF2. Mutating either of these two residues to an alanine not only impairs binding to m6A-containing 16mer RNA oligonucleotides but also abolishes the sequence preference of YTHDC1 at the -1 position. In contrast, the G-1 nucleotide only forms one hydrophobic interaction with Y397 in YTHDF1, which may result in decreased selectivity between YTHDF1 and the G-1 nucleotide (Fig. 4). In summary, compared to other YTH domains, the YTHDC1 YTH domain adopts a unique G-1 binding pocket, by which YTHDC1 acquires selectivity for the nucleotide preceding the m6A.

\section{YTH family proteins' roles in human cancer}

Since N6-methyladenosine modification affects gene expression during multiple steps of RNA metabolic processes, many studies have found that m6A is critical in many diseases, including cancer ${ }^{87,88}$. As important readers, YTH family proteins are involved in almost every aspect of tumorigenesis and cancer progression (Table 3).

\section{YTHDF1's role in human cancer}

As mentioned above, YTHDF1, a reader of m6A, can promote the translation of some transcripts to change the proteome in cancer cells, therefore regulating tumorigenesis. Additionally, several kinds of tumors have been reported to be related to YTHDF1. Many studies have indicated that YTHDF1 is an oncogene. For example, YTHDF1 is highly expressed in intestinal stem cells and colorectal cancer cells, and it participates in Wnt signaling. YTHDF1 can facilitate the translation of Wnt signaling effectors, including TCF7L2/TCF4, to augment $\beta$-catenin activity to regulate intestinal stem cell activity and tumorigenesis ${ }^{89,90}$. Similarly, in gastric cancer, mutated YTHDF1 enhances the translation of FZD7 to activate the Wnt- $\beta$-catenin pathway to promote gastric cancer cell proliferation and tumorigenesis ${ }^{91}$. In nonsmall cell lung cancer (NSCLC), YTHDF1 was reported to promote cancer cell proliferation and tumor progression by regulating the translational efficiency of CDK2, CDK4, and cyclin $\mathrm{D} 1^{92}$. In addition, YTHDF1 helps promote YAP mRNA translation in NSCLC, and the increases in YAP expression and activity induce drug resistance and metastasis in $\mathrm{NSCLC}^{93}$. Abnormally controlled translation of key mRNAs in the cancer genome and generally enhanced translational output are important responses to oncogenic stimulation $^{94}$. Indeed, in ovarian cancer, m6Amodified EIF3C, which is an essential initiation factor, is recognized and bound to YTHDF1. YTHDF1 promotes the translation of EIF3C and therefore enhances the total translational output, inducing cancer progression and 


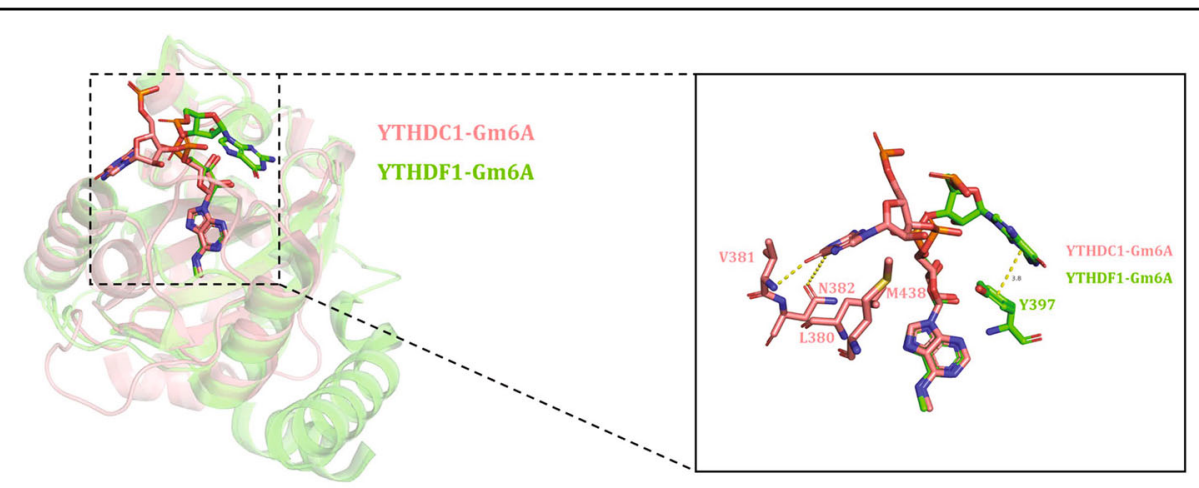

Fig. 4 Structural comparison of YTHDC1 and YTHDF1 reveals the structural basis for the discriminative recognition of the nucleotide preceding the m6A mark by YTHDC1. The YTHDC1-G(m6A) and YTHDF1-G(m6A) complexes are shown in the model as pink and green sticks, and the difference interactions between YTHDC1 and YTHDF1 with the G-1 nucleotide are highlighted with yellow dashed lines.

Table 3 List of the roles of YTH family proteins in different cancers.

\begin{tabular}{|c|c|c|c|}
\hline Cancer type or procedure & Reader & Main targets & Role \\
\hline \multirow[t]{2}{*}{ Colorectal cancer } & YTHDF1 & TCF4 & Oncogene \\
\hline & YTHDF3 & GAS5 & Oncogene \\
\hline \multirow[t]{3}{*}{ Hepatocellular carcinoma } & YTHDF2 & IL11, SERPINE2, EGFR & Tumor suppressor \\
\hline & YTHDF2 & OCT4 & Oncogene \\
\hline & YTHDF2 & socs2 & Mediator \\
\hline Pancreatic cancer & YTHDF2 & YAP signaling & Migration-proliferation dichotomy \\
\hline \multirow[t]{4}{*}{ Lung cancer } & YTHDF1 & CDK2, CDK4, cyclin D1, YAP & Oncogene \\
\hline & YTHDF2 & $6 \mathrm{PGD}$ & Oncogene \\
\hline & YTHDF3 & YAP & Hub of YTHDF1-2 \\
\hline & YTHDC2 & SLC7A11 & Tumor suppressor \\
\hline Gastric cancer & YTHDF1 & FZD7 & Oncogene \\
\hline Ovarian cancer & YTHDF1 & EIF3C & Oncogene \\
\hline Bladder cancer & YTHDF1 & ITGA6, CDCP1 & Oncogene \\
\hline Prostate cancer & YTHDF2 & LHPP, NKX3-1 & Oncogene \\
\hline Merkel cell carcinoma & YTHDF1 & Unknown & Oncogene \\
\hline Acute myeloid leukemia & YTHDF2 & Tnfrsf2 & Oncogene \\
\hline Epithelial-mesenchymal transition & YTHDF1 & Snail & Oncogene \\
\hline Antitumour immune response & YTHDF1 & Lysosomal proteases & Weaken antitumor response \\
\hline Ocular melanoma & YTHDF1 & HINT2 & Tumor suppressor \\
\hline \multirow[t]{2}{*}{ Glioblastoma } & YTHDF2 & MYC, VEGFA, LXRa, HIVEP2 & Oncogene \\
\hline & YTHDC1 & SRSF & Mediator \\
\hline Endometrial carcinoma & YTHDC1 & VEGF, BRCA1, PGR & Oncogene \\
\hline \multirow[t]{2}{*}{ Cancer metastasis } & YTHDF3 & ST6GALNAC5, GJA1, and EGFR & Oncogene \\
\hline & YTHDC2 & HIF-1a & Oncogene \\
\hline Nasopharyngeal carcinoma & YTHDC2 & IGF1R & Oncogene \\
\hline
\end{tabular}


metastasis $^{95}$. It was also reported that YTHDF1 has an important role in bladder cancer. YTHDF1 helps promote the translation of ITGA6 and CDCP1 mRNA, and high expression of these factors can increase the growth and progression of bladder cancer ${ }^{96,97}$. Merkel cell carcinoma is deadly skin cancer in which YTHDF1 was found to be highly expressed and associated with tumorigenesis ${ }^{98}$. Additionally, YTHDF1 has been implicated in epithelial-mesenchymal transition (EMT), of which the transcription factor Snail is known to be a critical regulator. YTHDF1 was reported to mediate the m6Aincreased translation of Snail mRNA and thus regulate EMT in cancer cells ${ }^{19}$. In the field of tumor immunotherapy, it was reported that durable neoantigenspecific immunity is suppressed by YTHDF1. Mechanistically, m6A-containing transcripts encoding lysosomal proteases are recognized by YTHDF1, and thus elevated expression at the translational level promotes the degradation of tumor neoantigens and represses crosspresentation to influence the efficacy of immunotherapy $^{99}$. Moreover, there is some evidence indicating that YTHDF1 is associated with poor prognosis in patients with hepatocellular carcinoma and breast cancer ${ }^{100-103}$. However, YTHDF1 was also found to act as a tumor suppressor by promoting the translation of the methylated mRNA of HINT2, a tumor suppressor in ocular melanoma ${ }^{104}$.

\section{YTHDF2's role in human cancer}

Another important reader, YTHDF2, is also well researched and has been found to be closely related to human cancer. Among the many different kinds of tumors, hepatocellular carcinoma (HCC) is one in which YTHDF2 has been studied the most. YTHDF2 can influence tumor progression in several different ways. YTHDF2 generally works as a tumor suppressor in HCC, as it mediates the decay of m6A-containing IL11 and SERPINE2 mRNAs, which are mediators of cancerpromoting inflammation and reprogramming of the tumor vasculature ${ }^{69}$. YTHDF2 also suppresses ERK/ MAPK signaling by destabilizing EGFR in an m6Adependent manner to inhibit the growth and proliferation of HCC cells ${ }^{105}$. Moreover, YTHDF2 participates in HCC progression in another way, although it does not have a core role. It binds SOCS2 mRNA and mediates its degradation to promote liver cancer progression, while METTL3 regulates the m6A level of SOCS2 mRNA ${ }^{106}$. It was reported that miR-145 targets the $3^{\prime}$ UTR of YTHDF2 mRNA, thus affecting the decay of m6A-containing mRNA to influence the m6A level in HCC cells. However, this research showed that YTHDF2 was closely associated with the malignancy of $\mathrm{HCC}^{107}$. Indeed, another research group regarded YTHDF2 as an oncogene in HCC because they found that YTHDF2 increased
OCT4 expression to promote liver cancer metas$\operatorname{tasis}^{108,109}$. Regarding other types of cancer, YTHDF2 is overexpressed in acute myeloid leukemia and is required for disease initiation. Mechanistically, YTHDF2 destabilizes m6A-modified transcripts such as that of the tumor necrosis factor receptor Tnfrsf 2 to protect self-renewing leukemic stem cells against apoptosis ${ }^{110}$. YTHDF2 promotes the proliferation and inhibits the migration and invasion as well as EMT of pancreatic cancer cells, probably through YAP signaling, although the exact mechanism remains to be clarified ${ }^{111}$. The oncogenicity of YTHDF2 was revealed in prostate cancer, and YTHDF2 mediates the degradation of LHPP and NKX3-1 to induce the phosphorylation of $\mathrm{AKT}^{112}$. In glioblastoma, in contrast to its proposed role, YTHDF2 was shown to stabilize MYC and VEGFA to maintain the oncogenic phenotype of glioblastoma stem cells ${ }^{113}$. It was also reported that YTHDF2 was phosphorylated at serine 39 and threonine 381 through EGFR/SRC/ERK signaling in glioblastoma ${ }^{114}$. The phosphorylation of YTHDF2 stabilized the YTHDF2 protein and promoted the decay of LXRA and HIVEP2 mRNA, which is required for cholesterol dysregulation, cell proliferation, invasion, and tumorigenesis in glioblastoma. Interestingly, although YTHDF2 was regarded to control mRNA decay, YTHDF2 was reported to facilitate 6PGD mRNA translation to promote lung cancer cell growth $^{115}$, similar to the role of YTHDF1 and the same effect as the increased OCT4 expression mentioned above $^{108}$. Notably, YTHDF2 has an important role in the regulatory effects of m6A methylases and demethylases on the tumorigenicity of osteosarcoma, breast tumors, melanoma, bladder cancer, pancreatic cancer, and colorectal cancer ${ }^{116-123}$.

\section{Other readers in human cancer}

The other three YTH family proteins, YTHDF3, YTHDC1, and YTHDC2, were found to be less correlated with human cancer than YTHDF1 and YTHDF2. Recently, YTHDF3 was found to be overexpressed and enhance the translation of ST6GALNAC5, GJA1, and EGFR to promote brain metastasis in breast cancer ${ }^{109}$. A negative functional loop constituted by the lncRNA GAS5-YAP-YTHDF3 axis was revealed in colorectal cancer, as GAS5 interacts with the WW domain of YAP to facilitate YAP shuttling from the nucleus to the cytoplasm and YAP phosphorylation; subsequently, YAP is degraded in a ubiquitin-mediated manner to inhibit CRC progression. Importantly, YTHDF3 is a target of YAP signaling and mediates the decay of m6A-modified GAS5 $\mathrm{mRNA}^{124}$. Regarding YTHDF3, research in NSCLC revealed the mechanism by which YTHDF3 acts as a hub to fine-tune the accessibility of RNA to YTHDF1 and $\mathrm{YTHDF}^{30}$. As demonstrated above, YAP mRNA is recognized by YTHDF3 and is then assigned to YTHDF1 
or YTHDF2 to be destabilized or translated; therefore, YTHDF3 is able to control YAP signaling to regulate cell proliferation, metastasis, and other tumorigenic behaviors. In addition, YTHDC1, which is an m6A reader involved in RNA splicing, was reported to recognize m6A modification around the start codon of serine/argininerich splicing factors (SRSFs) and lead to nonsensemediated mRNA decay, which affects the alternative splicing of a number of genes, such as BCL-X and NCOR2, eventually causing cancer-related phenotypes mediated by METTL3 in glioblastoma ${ }^{125}$. In addition, the ability of YTHDC1 to splice transcripts has been demonstrated for a vascular endothelial growth factor (VEGF), breast cancer 1 (BRCA1), and the progesterone receptor (PGR) in endometrial carcinoma, but whether these processes are dependent on m6A modification is still unclear ${ }^{126,127}$. YTHDC2, the last reader, was reported to be upregulated in human cancer cell lines and to promote cancer metastasis by promoting translation initiation via unwinding of the $5^{\prime}$-untranslated region $\left(5^{\prime}\right.$ UTR) of mRNAs such as HIF- $1 \alpha^{128}$. Moreover, YTHDC2 was found to be highly expressed in radioresistant nasopharyngeal carcinoma and to promote radioresistance by activating the IGF1R/ATK/S6 signaling axis ${ }^{129}$. YTHDC2 also regulates redox homeostasis and inhibits LUAD tumorigenesis since it promotes m6A-dependent mRNA degradation of SLC7A11, which is the core component of a cystine/glutamate antiporter ${ }^{130}$.

\section{Conclusions and perspectives}

In the early years, studies about the association between m6A modification and human cancer were centered around the balance of $\mathrm{m} 6 \mathrm{~A}$ addition and removal by writers and erasers, respectively ${ }^{6,131-133}$. However, changes in the readers could be a more crucial factor in the fate of RNAs. Therefore, we ask whether the switch from one reader to another could be a more reasonable strategy to control gene expression. Indeed, readers have recently been increasingly emphasized in epigenetic m6A modifications. Since domains other than the YTH domain of YTHDFs may only function after the binding process, it is likely that the YTH domain is responsible for recognizing target mRNAs. However, the targets of different readers have a certain degree of overlap, and the mechanism underlying the selectivity of readers is not well understood and may involve preferred motifs, phase separation, or the possible assigning function of YTHDF3 and other unknown factors. The upstream regulation of YTH family proteins is still unclear, although it has been found that miR-145 may affect YTHDF2 mRNA $^{107}$. It is worth noting that there is an emerging concept that YTHDF proteins are redundant in function and that their only effect is destabilizing transcripts, which makes this field more complicated. In different types of tumor cells, the specific regulatory mechanism of m6A differs. It is possible that the functions of different readers can partially overlap, and this possibility still needs to be further investigated. From the perspectives of translational medicine and clinical medicine, drugs targeting the YTH family may be a potential strategy for certain cancers. Although specific chemical inhibitors targeting the YTH domain have yet to be discovered, the above investigation of the structural biology of different YTH domains has paved the way for the rational design of small-molecule YTH domain inhibitors. Notably, through a recent virtual screen and crystallographic analysis, Rajiv et al. identified some promising hits as competitive YTH domain modulators that were expected to efficiently disrupt the interactions between $\mathrm{m} 6 \mathrm{~A}$ and YTHDC1. Specifically, the author indicated that $\mathrm{N}$-methyl amides could constitute appropriate fragments to compete with m6A molecules. Overall, considering the importance of the YTH family in cancer progression, the development of specific YTH domain inhibitors would not only enhance our knowledge of cancer epigenetics but also provide novel targeted therapies.

\section{Acknowledgements \\ We thank members of our laboratories for their suggestions and help.}

\section{Author contributions}

H.J., X.W., and R.S. designed the study. R.S. and S.Y. collected the related references, wrote the manuscript, and constructed the figures. H.J., R.S., S.Y., Y. L., and L.Z. revised the manuscript. All authors approved the final manuscript and agreed to be responsible for this review.

\section{Funding}

The authors received no specific funding for this work.

Ethics statement

No ethics approvals were required for this paper.

Conflict of interest

The authors declare no competing interests.

\section{Publisher's note}

Springer Nature remains neutral with regard to jurisdictional claims in published maps and institutional affiliations.

Received: 8 November 2020 Revised: 9 March 2021 Accepted: 15 March 2021

Published online: 01 April 2021

\footnotetext{
References

1. Desrosiers, R., Friderici, K. \& Rottman, F. Identification of methylated nucleosides in messenger RNA from Novikoff hepatoma cells. Proc. Natl Acad. Sci. USA 71, 3971-3975 (1974).

2. Csepany, T., Lin, A., Baldick, C. J. Jr \& Beemon, K. Sequence specificity of mRNA N6-adenosine methyltransferase. J. Biol. Chem. 265, 20117-20122 (1990).

3. Meyer, K. D. et al. Comprehensive analysis of mRNA methylation reveals enrichment in 3' UTRs and near stop codons. Cell 149, 1635-1646 (2012).

4. Dominissini, D. et al. Topology of the human and mouse m6A RNA methylomes revealed by m6A-seq. Nature 485, 201-206 (2012).
} 
5. Zhou, K. I. \& Pan, T. An additional class of m(6)A readers. Nat. Cell Biol. 20, 230-232 (2018).

6. Chen, X. Y., Zhang, J. \& Zhu, J. S. The role of m(6)A RNA methylation in human cancer. Mol. Cancer 18, 103 (2019).

7. Peritz, T. et al. Immunoprecipitation of mRNA-protein complexes. Nat. Protoc. 1. 577-580 (2006).

8. Ule, J. et al. CLIP identifies Nova-regulated RNA networks in the brain. Science 302, 1212-1215 (2003).

9. Hafner, M. et al. Transcriptome-wide identification of RNA-binding protein and microRNA target sites by PAR-CLIP. Cell 141, 129-141 (2010).

10. Wang, $X$. et al. N6-methyladenosine-dependent regulation of messenger RNA stability. Nature 505, 117-120 (2014).

11. Liu, J. et al. A METTL3-METTL14 complex mediates mammalian nuclear RNA N6-adenosine methylation. Nat. Chem. Biol. 10, 93-95 (2014).

12. Ping, $X$. L. et al. Mammalian WTAP is a regulatory subunit of the RNA N6methyladenosine methyltransferase. Cell Res. 24, 177-189 (2014).

13. Patil, D. P. et al. m(6)A RNA methylation promotes XIST-mediated transcriptional repression. Nature 537, 369-373 (2016).

14. Yue, Y. et al. VIRMA mediates preferential m(6)A mRNA methylation in $3^{\prime} U T R$ and near stop codon and associates with alternative polyadenylation. Cell Discov. 4, 10 (2018)

15. Wen, J. et al. Zc3h13 regulates nuclear RNA m(6)A methylation and mouse embryonic stem cell self-renewal. Mol. Cell 69, 1028-1038 (2018).

16. Jia, G. et al. N6-methyladenosine in nuclear RNA is a major substrate of the obesity-associated FTO. Nat. Chem. Biol. 7, 885-887 (2011).

17. Zheng, G. et al. ALKBH5 is a mammalian RNA demethylase that impacts RNA metabolism and mouse fertility. Mol. Cell 49, 18-29 (2013).

18. Wang, $X$. et al. N(6)-methyladenosine modulates messenger RNA translation efficiency. Cell 161, 1388-1399 (2015).

19. Lin, $X$. et al. RNA m(6)A methylation regulates the epithelial mesenchymal transition of cancer cells and translation of Snail. Nat. Commun. 10, 2065 (2019).

20. Zhao, W. et al. METTL3 facilitates oral squamous cell carcinoma tumorigenesis by enhancing c-Myc stability via YTHDF1-mediated m(6)A modification. Mol. Ther. Nucleic Acids 20, 1-12 (2020).

21. Lin, Z. et al. RNA m(6) A methylation regulates sorafenib resistance in liver cancer through FOXO3-mediated autophagy. EMBO J. 39, e103181 (2020).

22. Wang, Q. et al. N(6)-methyladenosine METTL3 promotes cervical cancer tumorigenesis and Warburg effect through YTHDF1/HK2 modification. Cell Death Dis. 11, 911 (2020).

23. Weng, $Y$. L. et al. Epitranscriptomic $m(6) A$ regulation of axon regeneration in the adult mammalian nervous system. Neuron 97, 313-325 (2018).

24. Zhuang, M. R. et al. The $\mathrm{m}(6) \mathrm{A}$ reader $\mathrm{YTHDF1}$ regulates axon guidance through translational control of Robo3.1 expression. Nucleic Acids Res. 47, 4765-4777 (2019).

25. Shi, H. L. et al. m(6)A facilitates hippocampus-dependent learning and memory through YTHDF1. Nature 563, 249 (2018).

26. $\mathrm{Du}, \mathrm{H}$. et al. YTHDF2 destabilizes $\mathrm{m}(6) \mathrm{A}$-containing RNA through direct recruitment of the CCR4-NOT deadenylase complex. Nat. Commun. 7, 12626 (2016).

27. Zhao, B. S. et al. m(6)A-dependent maternal mRNA clearance facilitates zebrafish maternal-to-zygotic transition. Nature 542, 475-478 (2017).

28. Park, O. H. et al. Endoribonucleolytic cleavage of m(6)A-containing RNAs by RNase P/MRP complex. Mol. Cell 74, 494-507.e8 (2019).

29. Shi, H. et al. YTHDF3 facilitates translation and decay of N(6)-methyladenosine-modified RNA. Cell Res. 27, 315-328 (2017).

30. Jin, D. et al. m(6)A demethylase ALKBH5 inhibits tumor growth and metastasis by reducing YTHDFs-mediated YAP expression and inhibiting miR-107/LATS2-mediated YAP activity in NSCLC. Mol. Cancer 19, 40 (2020).

31. Ries, R. J. et al. m(6)A enhances the phase separation potential of mRNA. Nature 571, 424-428 (2019).

32. Gao, Y. et al. Multivalent m(6)A motifs promote phase separation of YTHDF proteins. Cell Res. 29, 767-769 (2019).

33. Wang, J. et al. Binding to m(6)A RNA promotes YTHDF2-mediated phase separation. Protein Cell 11, 304-307 (2020).

34. Fu, Y. \& Zhuang, X. m(6)A-binding YTHDF proteins promote stress granule formation. Nat. Chem. Biol. 16, 955-963 (2020).

35. Zhou, J. et al. Dynamic m(6)A mRNA methylation directs translational control of heat shock response. Nature 526, 591-594 (2015).

36. Panneerdoss, S. et al. Cross-talk among writers, readers, and erasers of m(6)A regulates cancer growth and progression. Sci. Adv. 4, eaar8263 (2018).
37. Song, T. et al. Zfp217 mediates m6A mRNA methylation to orchestrate transcriptional and post-transcriptional regulation to promote adipogenic differentiation. Nucleic Acids Res. 47, 6130-6144 (2019).

38. Zaccara, S. \& Jaffrey, S. R. A unified model for the function of YTHDF proteins in regulating m(6)A-modified mRNA. Cell 181, 1582-1595 (2020).

39. Lasman, L. et al. Context-dependent functional compensation between Ythdf m(6)A reader proteins. Genes Dev. 34, 1373-1391 (2020).

40. Li, Y., Bedi, R. K., Moroz-Omori, E. V. \& Caflisch, A. Structural and dynamic insights into redundant function of YTHDF proteins. J. Chem. Inf. Model 60 5932-5935 (2020).

41. Imai, Y., Matsuo, N., Ogawa, S., Tohyama, M. \& Takagi, T. Cloning of a gene, YT521, for a novel RNA splicing-related protein induced by hypoxia/reoxygenation. Brain Res. Mol. Brain Res. 53, 33-40 (1998).

42. Nayler, O., Hartmann, A. M. \& Stamm, S. The ER repeat protein YT521-B localizes to a novel subnuclear compartment. J. Cell Biol. 150, 949-962 (2000).

43. Hartmann, A. M., Nayler, O., Schwaiger, F. W., Obermeier, A. \& Stamm, S. The interaction and colocalization of Sam68 with the splicing-associated factor YT521-B in nuclear dots is regulated by the Src family kinase p59(fyn). Mol. Biol. Cell 10, 3909-3926 (1999).

44. Rafalska, I. et al. The intranuclear localization and function of YT521-B is regulated by tyrosine phosphorylation. Hum. Mol. Genet. 13, 1535-1549 (2004).

45. Xiao, W. et al. Nuclear $m(6) A$ reader YTHDC1 regulates mRNA splicing. Mol. Cell 61, 507-519 (2016)

46. Roundtree, I. A. et al. YTHDC1 mediates nuclear export of N(6)-methyladenosine methylated mRNAs. Elife 6, e31311 (2017).

47. Haussmann, I. U. et al. m(6)A potentiates Sxl alternative pre-mRNA splicing for robust Drosophila sex determination. Nature 540, 301-304 (2016).

48. Lence, T. et al. m(6)A modulates neuronal functions and sex determination in Drosophila. Nature 540, 242-247 (2016).

49. Liu, J. et al. $\mathrm{N}$ (6)-methyladenosine of chromosome-associated regulatory RNA regulates chromatin state and transcription. Science 367, 580-586 (2020).

50. Shima, H. et al. S-adenosylmethionine synthesis is regulated by selective N(6)adenosine methylation and mRNA degradation Involving METTL16 and YTHDC1. Cell Rep. 21, 3354-3363 (2017).

51. Li, Y. et al. N(6)-methyladenosine co-transcriptionally directs the demethylation of histone H3K9me2. Nat. Genet. 52, 870-877 (2020).

52. Morohashi, K. et al. Cyclosporin A associated helicase-like protein facilitates the association of hepatitis C virus RNA polymerase with its cellular cyclophilin B. PLOS ONE 6, e18285 (2011)

53. Hsu, P. J. et al. Ythdc2 is an N(6)-methyladenosine binding protein that regulates mammalian spermatogenesis. Cell Res. 27, 1115-1127 (2017).

54. Wojtas, M. N. et al. Regulation of m(6)A transcripts by the $3^{\prime}->5^{\prime}$ RNA helicase YTHDC2 is essential for a successful meiotic program in the mammalian germline. Mol. Cell 68, 374-387 (2017).

55. Abby, E. et al. Implementation of meiosis prophase I programme requires a conserved retinoid-independent stabilizer of meiotic transcripts. Nat. Commun. 7, 10324 (2016).

56. Soh, Y. Q. S. et al. Meioc maintains an extended meiotic prophase I in mice. PLoS Genet. 13, e1006704 (2017).

57. Bailey, A. S. et al. The conserved RNA helicase YTHDC2 regulates the transition from proliferation to differentiation in the germline. Elife 6, e26116 (2017).

58. Jain, D. et al. ketu mutant mice uncover an essential meiotic function for the ancient RNA helicase YTHDC2. Elife 7, e30919 (2018).

59. Kretschmer, J. et al. The $\mathrm{m}(6) \mathrm{A}$ reader protein $\mathrm{YTHDC2}$ interacts with the small ribosomal subunit and the 5'-3' exoribonuclease XRN1. RNA 24, 1339-1350 (2018).

60. Mao, Y. et al. m(6)A in mRNA coding regions promotes translation via the RNA helicase-containing YTHDC2. Nat. Commun. 10, 5332 (2019).

61. Nakano, M., Ondo, K, Takemoto, S., Fukami, T. \& Nakajima, M. Methylation of adenosine at the $\mathrm{N}(6)$ position post-transcriptionally regulates hepatic P450s expression. Biochem Pharm. 171, 113697 (2020).

62. Zhou, B. et al. N(6) -Methyladenosine Reader Protein YT521-B Homology Domain-Containing 2 Suppresses Liver Steatosis by Regulation of mRNA Stability of Lipogenic Genes. Hepatology 73, 91-103 (2021).

63. Stoilov, P., Rafalska, I. \& Stamm, S. YTH: a new domain in nuclear proteins. Trends Biochem. Sci. 27, 495-497 (2002).

64. Zhang, Z. et al. The YTH domain is a novel RNA binding domain. J. Biol. Chem. 285, 14701-14710 (2010). 
65. Harigaya, Y. et al. Selective elimination of messenger RNA prevents an incidence of untimely meiosis. Nature 442, 45-50 (2006).

66. Kang, H. J. et al. A novel protein, Pho92, has a conserved YTH domain and regulates phosphate metabolism by decreasing the mRNA stability of $\mathrm{PHO} 4$ in Saccharomyces cerevisiae. Biochem. J. 457, 391-400 (2014).

67. Baumgarten, S. et al. Transcriptome-wide dynamics of extensive m(6)A mRNA methylation during Plasmodium falciparum blood-stage development. Nat. Microbiol. 4, 2246-2259 (2019).

68. Li, D. Y. et al. Genome-wide identification, biochemical characterization, and expression analyses of the YTH domain-containing RNA-binding protein family in Arabidopsis and rice. Plant Mol. Biol. Rep. 32, 1169-1186 (2014).

69. Wang, N., Yue, Z., Liang, D. \& Ma, F. Genome-wide identification of members in the YTH domain-containing RNA-binding protein family in apple and expression analysis of their responsiveness to senescence and abiotic stresses. Gene 538, 292-305 (2014).

70. Sun, J., Bie, X. M., Wang, N., Zhang, X. S. \& Gao, X. Q. Genome-wide identification and expression analysis of $\mathrm{YTH}$ domain-containing RNA-binding protein family in common wheat. BMC Plant Biol. 20, 351 (2020).

71. Govindaraju, G. et al. 6-Adenosine methylation on mRNA is recognized by YTH2 domain protein of human malaria parasite Plasmodium falciparum. Epigenet. Chromatin 13, 33 (2020).

72. Arribas-Hernandez, L. et al. An m(6)A-YTH module controls developmental timing and morphogenesis in Arabidopsis. Plant Cell 30, 952-967 (2018).

73. Arribas-Hernandez, L. et al. Recurrent requirement for the m(6)A-ECT2/ECT3/ ECT4 axis in the control of cell proliferation during plant organogenesis. Development 147, dev189134 (2020).

74. Yamashita, A. et al. Hexanucleotide motifs mediate recruitment of the RNA elimination machinery to silent meiotic genes. Open Biol. 2, 120014 (2012).

75. Zofall, M. et al. RNA elimination machinery targeting meiotic mRNAs promotes facultative heterochromatin formation. Science 335, 96-100 (2012).

76. Tashiro, S., Asano, T., Kanoh, J. \& Ishikawa, F. Transcription-induced chromatin association of RNA surveillance factors mediates facultative heterochromatin formation in fission yeast. Genes Cells 18, 327-339 (2013).

77. Hiriart, E. et al. Mmi1 RNA surveillance machinery directs RNAi complex RITS to specific meiotic genes in fission yeast. EMBO J. 31, 2296-2308 (2012).

78. Vo, T. V. et al. CPF recruitment to non-canonical transcription termination sites triggers heterochromatin assembly and gene silencing. Cell Rep. 28, 267-281 (2019).

79. $\mathrm{Xu}, \mathrm{C}$. et al. Structural basis for selective binding of m6A RNA by the YTHDC1 YTH domain. Nat. Chem. Biol. 10, 927-929 (2014).

80. Theler, D., Dominguez, C., Blatter, M., Boudet, J. \& Allain, F. H. Solution structure of the YTH domain in complex with N6-methyladenosine RNA: a reader of methylated RNA. Nucleic Acids Res. 42, 13911-13919 (2014).

81. Luo, S. \& Tong, L. Molecular basis for the recognition of methylated adenines in RNA by the eukaryotic YTH domain. Proc. Natl Acad. Sci. USA 111, 13834-13839 (2014).

82. $\mathrm{Xu}, \mathrm{C}$. et al. Structural basis for the discriminative recognition of N6methyladenosine RNA by the human YT521-B homology domain family of proteins. J. Biol. Chem. 290, 24902-24913 (2015).

83. Ma, C., Liao, S. \& Zhu, Z. Crystal structure of human YTHDC2 YTH domain. Biochem. Biophys. Res. Commun. 518, 678-684 (2019).

84. Li, F., Zhao, D., Wu, J. \& Shi, Y. Structure of the YTH domain of human YTHDF2 in complex with an $\mathrm{m}(6) \mathrm{A}$ mononucleotide reveals an aromatic cage for $\mathrm{m}$ (6)A recognition. Cell Res. 24, 1490-1492 (2014).

85. Zhu, T. et al. Crystal structure of the YTH domain of YTHDF2 reveals mechanism for recognition of N6-methyladenosine. Cell Res. 24, 1493-1496 (2014).

86. Wei, C. M. \& Moss, B. Nucleotide sequences at the N6-methyladenosine sites of HeLa cell messenger ribonucleic acid. Biochemistry 16, 1672-1676 (1977).

87. He, L. et al. Functions of N6-methyladenosine and its role in cancer. Mol. Cancer 18, 176 (2019).

88. Huang, H., Weng, H. \& Chen, J. m(6)A modification in coding and noncoding RNAs: roles and therapeutic implications in cancer. Cancer Cell $\mathbf{3 7}$ 270-288 (2020).

89. Bai, Y. et al. YTHDF1 regulates tumorigenicity and cancer stem cell-like activity in human colorectal carcinoma. Front. Oncol. 9, 332 (2019).

90. Han, B. et al. YTHDF1-mediated translation amplifies Wnt-driven intestinal stemness. EMBO Rep. 21, e49229 (2020).
91. Pi, J. et al. YTHDF1 promotes gastric carcinogenesis by controlling translation of FZD7. Cancer Res. (2020).

92. Shi, Y. et al. YTHDF1 links hypoxia adaptation and non-small cell lung cancer progression. Nat. Commun. 10, 4892 (2019).

93. Jin, D. et al. m(6)A mRNA methylation initiated by METTL3 directly promotes YAP translation and increases YAP activity by regulating the MALAT1-miR1914-3p-YAP axis to induce NSCLC drug resistance and metastasis. J. Hematol. Oncol. 12, 135 (2019).

94. Truitt, M. L. \& Ruggero, D. New frontiers in translational control of the cancer genome. Nat. Rev. Cancer 16, 288-304 (2016).

95. Liu, $\mathrm{T}$. et al. The $\mathrm{m} 6 \mathrm{~A}$ reader $\mathrm{YTHDF1}$ promotes ovarian cancer progression via augmenting EIF3C translation. Nucleic Acids Res. 48, 3816-3831 (2020).

96. Jin, $\mathrm{H}$. et al. N(6)-methyladenosine modification of ITGA6 mRNA promotes the development and progression of bladder cancer. EBioMedicine 47, 195-207 (2019).

97. Yang, F. et al. Dynamic m(6)A mRNA methylation reveals the role of METTL3$\mathrm{m}(6) \mathrm{A}-\mathrm{CDCP} 1$ signaling axis in chemical carcinogenesis. Oncogene $\mathbf{3 8}$, 4755-4772 (2019).

98. Orouji, E., Peitsch, W. K., Orouji, A., Houben, R. \& Utikal, J. Oncogenic role of an epigenetic reader of m(6)A RNA modification: YTHDF1 in merkel cell carcinoma. Cancers 12, 202 (2020).

99. Han, D. L. et al. Anti-tumour immunity controlled through mRNA m(6)A methylation and YTHDF1 in dendritic cells. Nature 566, 270 (2019).

100. Zhao, X. G. et al. Overexpression of YTHDF1 is associated with poor prognosis in patients with hepatocellular carcinoma. Cancer Biomark. 21, 859-868 (2018).

101. Zhou, Y. et al. Expression profiles and prognostic significance of RNA N6methyladenosine-related genes in patients with hepatocellular carcinoma: evidence from independent datasets. Cancer Manag. Res. 11, 3921-3931 (2019).

102. Liu, L. et al. N6-methyladenosine-related genomic targets are altered in breast cancer tissue and associated with poor survival. J. Cancer 10, 5447-5459 (2019).

103. Qu, N. et al. Multiple m(6)A RNA methylation modulators promote the malignant progression of hepatocellular carcinoma and affect its clinical prognosis. BMC Cancer 20, 165 (2020).

104. Jia, R. et al. m(6)A modification suppresses ocular melanoma through modulating HINT2 mRNA translation. Mol. Cancer 18, 161 (2019).

105. Zhong, L. et al. YTHDF2 suppresses cell proliferation and growth via destabilizing the EGFR mRNA in hepatocellular carcinoma. Cancer Lett. 442, 252-261 (2019).

106. Chen, M. et al. RNA N6-methyladenosine methyltransferase-like 3 promotes liver cancer progression through YTHDF2-dependent posttranscriptional silencing of SOCS2. Hepatology 67, 2254-2270 (2018).

107. Yang, Z. et al. MicroRNA-145 modulates N(6)-methyladenosine levels by targeting the 3 '-untranslated mRNA region of the N(6)-methyladenosine binding YTH domain family 2 protein. J. Biol. Chem. 292, 3614-3623 (2017).

108. Zhang, C. et al. YTHDF2 promotes the liver cancer stem cell phenotype and cancer metastasis by regulating OCT4 expression via m6A RNA methylation. Oncogene 39, 4507-4518 (2020).

109. Chang, $G$. et al. YTHDF3 induces the translation of $m(6)$ A-enriched gene transcripts to promote breast cancer brain metastasis. Cancer Cell $\mathbf{3 8}$, 857-871 e857 (2020).

110. Paris, J. et al. Targeting the RNA m(6)A reader YTHDF2 selectively compromises cancer stem cells in acute myeloid leukemia. Cell Stem Cell 25, 137-148 (2019).

111. Chen, J. et al. YTH domain family 2 orchestrates epithelial-mesenchymal transition/proliferation dichotomy in pancreatic cancer cells. Cell Cycle 16, 2259-2271 (2017)

112. Li, J. et al. YTHDF2 mediates the mRNA degradation of the tumor suppressors to induce AKT phosphorylation in N6-methyladenosine-dependent way in prostate cancer. Mol. Cancer 19, 152 (2020).

113. Dixit, D. et al. The RNA m6A Reader YTHDF2 Maintains Oncogene Expression and Is a Targetable Dependency in Glioblastoma Stem Cells. Cancer Discov 11, 480-499 (2020).

114. Fang, R. et al. EGFR/SRC/ERK-stabilized YTHDF2 promotes cholesterol dysregulation and invasive growth of glioblastoma. Nat. Commun. 12, 177 (2021).

115. Sheng, E. et al. YTH domain family 2 promotes lung cancer cell growth by facilitating 6-phosphogluconate dehydrogenase mRNA translation. Carcinogenesis 41, 541-550 (2019). 
116. Chen, S., Zhou, L. \& Wang, Y. ALKBH5-mediated m(6)A demethylation of IncRNA PVT1 plays an oncogenic role in osteosarcoma. Cancer Cell Int 20, 34 (2020).

117. Niu, Y. et al. RNA N6-methyladenosine demethylase FTO promotes breast tumor progression through inhibiting BNIP3. Mol. Cancer 18, 46 (2019).

118. Yang, S. et al. m(6)A mRNA demethylase FTO regulates melanoma tumorigenicity and response to anti-PD-1 blockade. Nat. Commun. 10, 2782 (2019).

119. Xie, $H$. et al. METTL3/YTHDF2 m(6) A axis promotes tumorigenesis by degrading SETD7 and KLF4 mRNAs in bladder cancer. J. Cell Mol. Med. 24, 4092-4104 (2020).

120. Yang, X. et al. MEITL14 suppresses proliferation and metastasis of colorectal cancer by down-regulating oncogenic long non-coding RNA XIST. Mol. Cancer 19, 46 (2020).

121. Tian, J. et al. N(6)-methyladenosine mRNA methylation of PIK3CB regulates AKT signalling to promote PTEN-deficient pancreatic cancer progression. Gut 69, 2180-2192 (2020)

122. Chen, X. et al. METTL14-mediated N6-methyladenosine modification of SOX4 mRNA inhibits tumor metastasis in colorectal cancer. Mol. Cancer 19, 106 (2020)

123. Guo, X. et al. RNA demethylase ALKBH5 prevents pancreatic cancer progression by posttranscriptional activation of PER1 in an m6A-YTHDF2dependent manner. Mol. Cancer 19, 91 (2020).

124. Ni, W. et al. Long noncoding RNA GAS5 inhibits progression of colorectal cancer by interacting with and triggering YAP phosphorylation and degradation and is negatively regulated by the $\mathrm{m}(6) \mathrm{A}$ reader YTHDF3. Mol. Cancer 18, 143 (2019).
125. Li, F. et al. N(6)-methyladenosine modulates nonsense-mediated mRNA decay in human glioblastoma. Cancer Res. 79, 5785-5798 (2019).

126. Zhang, B. et al. YT521 promotes metastases of endometrial cancer by differential splicing of vascular endothelial growth factor A. Tumour Biol. 37, 15543-15549 (2015).

127. Hirschfeld, M. et al. Hypoxia-dependent mRNA expression pattern of splicing factor YT521 and its impact on oncological important target gene expression. Mol. Carcinog. 53, 883-892 (2014).

128. Tanabe, A. et al. RNA helicase YTHDC2 promotes cancer metastasis via the enhancement of the efficiency by which HIF-1alpha mRNA is translated. Cancer Lett. 376, 34-42 (2016).

129. He, J. J. et al. m(6)A reader YTHDC2 promotes radiotherapy resistance of nasopharyngeal carcinoma via activating IGF1R/AKT/S6 signaling axis. Front. Oncol. 10, 1166 (2020).

130. Ma, L. et al. The $\mathrm{m}(6) \mathrm{A}$ reader $\mathrm{YTHDC} 2$ inhibits lung adenocarcinoma tumorigenesis by suppressing SLC7A11-dependent antioxidant function. Redox Biol. 38, 101801 (2021).

131. Weng, $H$. et al. METTL14 inhibits hematopoietic stem/progenitor differentiation and promotes leukemogenesis via mRNA m(6)A modification. Cell Stem Cell 22, 191-205 (2018).

132. Huang, Y. et al. Small-molecule targeting of oncogenic FTO demethylase in acute myeloid leukemia. Cancer Cell 35, 677-691 (2019).

133. Cheng, $M$. et al. The $\mathrm{m}(6) \mathrm{A}$ methyltransferase METTL3 promotes bladder cancer progression via AFF4/NF-kappaB/MYC signaling network. Oncogene 38, 3667-3680 (2019). 